\title{
An Endocrinologic-Inspired Hardware Implementation of a Multicellular System
}

\author{
Andrew J. Greensted and Andy M. Tyrrell \\ Department of Electronics, \\ Intelligent Systems Research Group, \\ University of York, UK \\ YO10 5DD \\ tel: +441904432379 \\ http://www.bioinspired.com \\ E-mail: $\{a j g 112$, amt $\} @ o h m \cdot y o r k . a c . u k$
}

\begin{abstract}
Within higher animals there operates an inter-cell communication system that is responsible for regulating the physiological balance of its host. The endocrine system uses hormone mediated messages to control the function of remote cell groups, invoking reactions to maintain chemical and physical equilibrium. The operation and structure of the endocrine system exhibits a robustness and fault tolerance that has inspired the creation of a reliability engineered electronic architecture.

Previous work established a software model of such a system. The model was able to simulate arbitrary processing of a data stream whilst demonstrating tolerance to faults and repair. Following from this work, this paper outlines improvements to this model and the initial steps of its conversion into a hardware electronic system.

The Bionode System contains thirty individual nodes connected in a loosely coupled network. Each Bionode contains a microcontroller and FPGA that can be configured to model the functionality of a cell; specifically the underlying endocrine based communication system and the toplevel functions that perform useful data processing operations.
\end{abstract}

\section{Introduction}

The importance of redundancy in reliability engineered systems is unquestionable. A continually online system, whether it electronic, mechanical or biological, expected to exhibit tolerance of internal faults must make use of redundancy. Doing so in the correct manner increases the chances of such a system being able to circumvent a fault.
However, the organisation and control of redundancy is key to the success of any reliability scheme. A system where redundancy is badly distributed or proportioned, will be less able to allocate the required resources in an effective manner. In fact providing tolerance to faults, and recovery from them, puts two separate demands on redundancy. Tolerance demands that faults be masked when they occur, which requires additional resources in continuous use. Recoverability however requires the availability of spare dormant hardware to replace faulty sections.

The classic solution to these demands is a hybrid redundancy scheme [4]. This solution in its most basic form however requires considerable replication of whole systems, and can therefore be both costly and wasteful of resources. An answer to this problem is to increase the reliability scheme granularity. Utilising redundancy in smaller subsystems can localise the spread of the effects caused by faults, therefore potentially smaller amounts of resources need be lost at the occurrence of a fault.

A further step to improve the effectiveness of redundancy is increasing system homogeneity. The task of allocating resources to replace faulty system sections is greatly simplified if a generic slave unit can be used throughout the whole system.

A number of system characteristics have been highlighted for an improved reliability architecture. Inspiration from biology offers a rich source of ideas to develop such a scheme that expresses them. At the cellular level, higher animals ${ }^{1}$ exhibit extraordinary redundancy. The organisation and operation of which enables animals to suffer cell loss, and replenish the deficit left. Cellular biology has inspired

1 Higher animals refers to mammals or other vertebrates with advanced characteristics. 
a number of electronic architectures that attempt to exhibit fault tolerance and recovery [10][7]. This paper presents a novel multiprocessor architecture that exhibits these same properties, but with improved redundancy granularity and increased system function capacity.

The inter-node communication system of the architecture is based on endocrinology, a set of hormone mediated biological processes that allows cells to communicate. It is this communication system that allows faults within cells to be masked, system layout to be reconfigured and failed cells to be physically removed and replaced online.

The fault tolerance of hormone communcation has inspired other quite different work, such as the distributed control of robots [8]. However the work presented in this paper has been inspired by delving further into the biological processes that constitute the endocrine system.

Section 2 gives a brief explanation of endocrinology, highlighting the features that make it a robust system. Following, in section 3 , is a discussion of how endocrinology can inspire a reliability engineered system, and the development of a endocrinologic model suitable for electronic implementation. Section 4 details the Bionode system; the multiprocessor hardware platform on which the endocrinology inspired model runs. The paper ends with a conclusion and suggestions for further work, sections 5 and 6 respectively.

\section{Endocrinology}

There exist two fundamental categories of biological cell communication. The first, neurotransmission, operates directly over 'wired' routes. Electrical signals from the source propagate towards their target, on arrival the signal is converted to chemical messengers, and the final short step is made to the target [5]. The second category, endocrinology, utilises messengers that are far freer in their movements. It is this form of communication that is of interest to this work.

The messengers used by the endocrine system are hormones. They are able to travel around the body via the bloodstream, see figure 1. Communication involves a number of cells working together. A message starts by a group of collaborating cells releasing hormones into the bloodstream. These hormones have a set signature that only the desired target cells will respond to. If a cell bears receptors that have the correct affinity to the hormone signature, the two will bind completing the communication. There are further processes that take place internal to the cell, but within the context of this work, they are not important.

Hormones are able to invoke both stimulatory and inhibitory reactions in their target. In a single release of hormones separate groups of cells may be targeted, some being stimulated, and others inhibited. This enables complex control operations to be performed. Figure 2 shows a typi-

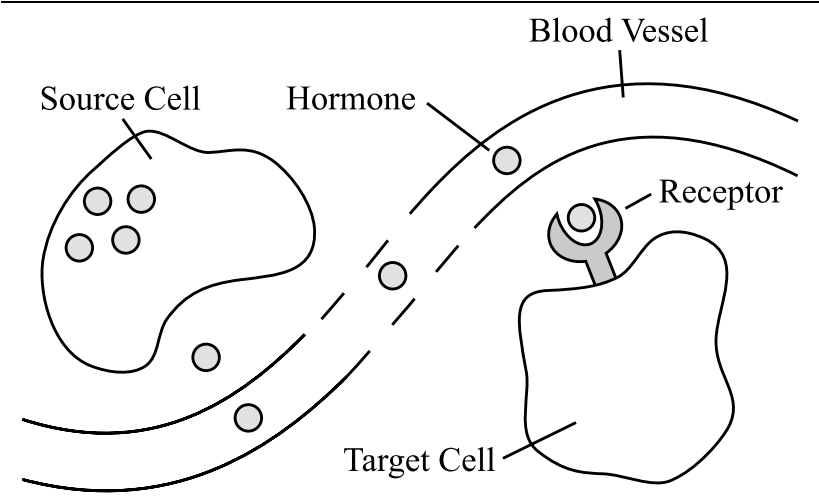

Figure 1. Endocrine Communcation, hormones that enter the blood stream are able to travel long distances to reach their target cells.

cal control scenario. The initial source cells release the first group of hormones. On reaching their target they invoke the creation of a secondary set of hormones that go on to perform two tasks. Firstly, they inhibit the original sources' production of hormones. This has the effect of confirming the arrival of the first hormone group. Secondly, they stimulate the next cell target group. This process is then repeated for each step. Overall, a simple negative feedback system is formed.

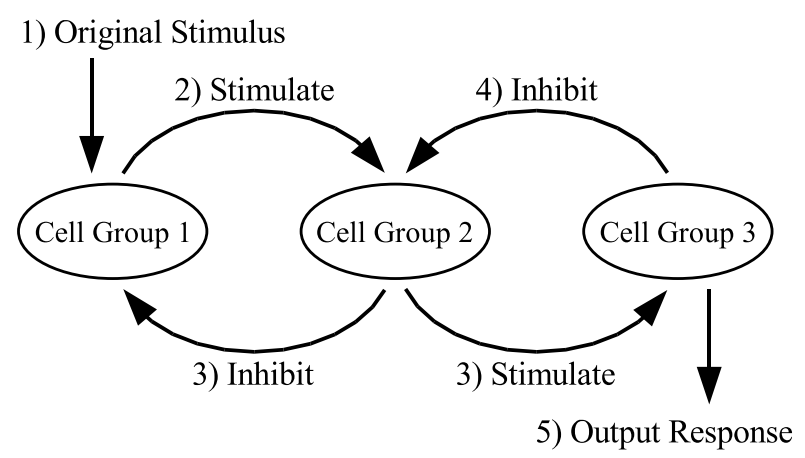

Figure 2. A cascade of hormone mediated cell reactions showing the negative feedback paths that help ensure the success of intercell communication.

Two other related communication methods are grouped within endocrinology. These are paracrine and autocrine communication, figure 3 . These generally perform positive feedback tasks, to help reinforce reception strength and the effect of messages. 


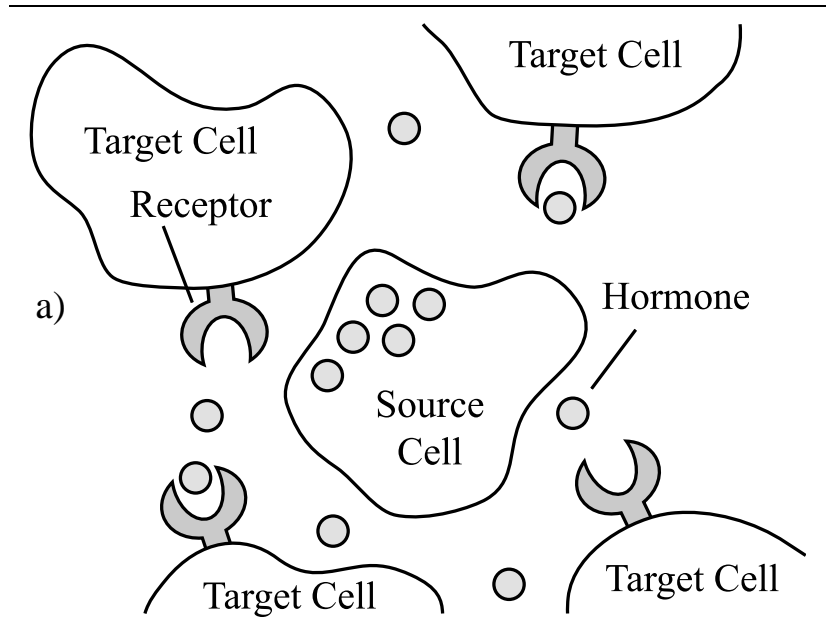

b)

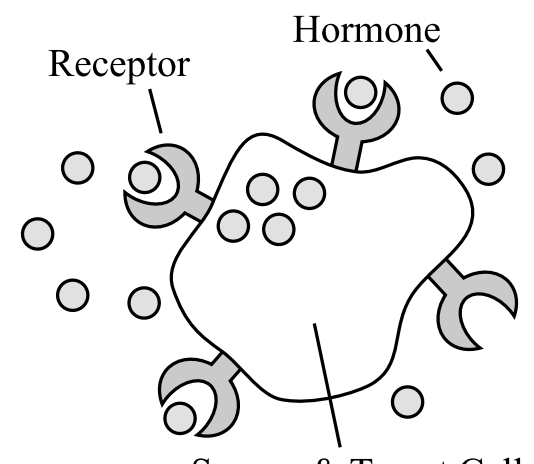

Source \& Target Cell

Figure 3. a) Paracrine communication involves distribution of hormones over and reduced local area. b) Autocrine communication allows a cell to communicate with itself.

\subsection{Endocrinology Reliability}

The endocrine system continually maintains homeostasis of animals in real-time. This means it provides physiological balance by controlling the chemical and physical composition of the animal [1]. This is achieved using a robust architecture tolerant of faults and appropriate for maintenance and repair. The most important features of the system that enable this are:

- Co-operating source and destination cell groups. Due to the large number of cells that co-operate to perform endocrine communication, in the event of small quantity cell loss the success of the communication task is not compromised. This has the additional benefit that cells may be easily removed and replaced whilst the system is operating without causing disruption.

- Threshold based cell reaction trigger. A number of specific hormones must bind to a cell before a reaction is invoked. Within the scales and quantities that biol- ogy operates, this means faulty hormones, or hormones released from faulty cells will be swamped amongst their fault-free companions. These masked minority of faulty hormones are therefore very unlikely to invoke a cell reaction.

- High specificity Hormone-Receptor binding. The binding mechanism between hormones and cells, similar to that of antibodies and antigen of the immune system [3], enables cells to distinguish which hormones are destined for it. Hormones are therefore free to coexist within the same migration space. As communication is not dependant on direct connections, a blood flow blockage does not stop hormones reaching their target as they are free to take alternative routes.

- Hormone reception acknowledgement. As described earlier, cells continue to release activating hormones until they receive inhibitory messages that confirm their arrival. This feedback system is far more reliable than simply releasing a fixed number of hormones and hoping they reach their target. The ability of single hormones to induce both stimulatory and inhibitory effects is more efficient than having to use two separate hormone types.

\section{Developing the Endocrinology Model for Electronics}

With knowledge of its operation, endocrinology provides an obvious source of inspiration for a reliability architecture. However as is generally the case with all cross discipline studies the mapping of biology to electronics is not straight forward.

The two fundamental difficulties are those of scale and structural medium. Per unit area, biological cells are able to perform more complex tasks than electronic circuitry. Also biological tissues have the luxury of dynamic structure. Parts may freely move, be removed and replaced. This is in considerable contrast with the currently static nature of electronic structures where circuitry positions are fixed.

With respect to creating an endocrine system model for electronics, these problems mean cell quantities must be considerably less, and hormone messengers must take the form of transmittable data rather than free moving physical matter. Another conversion hurdle, is the initial usefulness of the endocrine system to process data. The endocrine system does a good job of controlling physiological balance, but it is not directly capable of performing operations on arbitrary information. Details of how these issues were initially tackled to produce a useful endocrine system model can be found in [2]. Further explanation with details of improvements to the model are given in the remainder of this section. 


\subsection{Performing Data Processing}

The groups in which cells operate to perform the intercell communication of the endocrine system, neatly divides a mass of cells into sections. Furthermore, the cascade of hormone mediated reactions that pass from one group to another virtually connects the groups into a chain of consecutively activated stages. By considering each cell group as a separate processing unit, a chain of groups becomes a complete processing platform capable of performing operations on a flow of data through the system.

By including data in hormone messages, each cell group is able to perform an operation on the data and then send the result onto the next stage. The system input and output are respectively the start and end of the endocrine hormone cascade. Overall, the robustness of the underlying endocrine inspired architecture ensures the reliability of the complete processing platform.

Furthermore, as the hormone messages are only used as couriers of data, no constraints are placed on the complexity of the processing tasks performed by each cell.

\subsection{Dealing with Scale}

Biological systems utilise vast quantities of cells. The human brain requires millions of neurons to provide useful functionality, the immune system equally requires many cells to perform detection and neutralisation of pathogenic material. Similarly the endocrine system incorporates quantities of cells and hormones that are far beyond what currently may be realistically implemented in electronic hardware.

By requiring a number of hormones to bind before a reaction is invoked, a cell is able to mask the effects of faulty hormones. If however the number of hormones utilised in a endocrinologic model is reduced, the ratio of potentially faulty to fault-free hormones decreases. Therefore in the event of faults occurring, it is more probable that a stream of faulty hormones would be able to instigate an incorrect cell reaction. In addition, in a system that uses less hormones, the threshold of received hormones required to invoke a reaction would also have to be decreased, making it easier still for faulty hormones to disrupt the system.

The solution to this problem is to include a voting stage in the cell activation process. Instead of requiring a certain quantity of bound hormones to invoke a reaction, a number of hormones from differing cell sources are required. A masking effect is still achieved, but cell reactions can be triggered with far fewer hormone messages.

\subsection{Concurrent Data Processing}

The dynamics of the endocrine system are very much slower than is suitable for a useful data processing platform. The fluctuations in cell activity occur gradually enough to allow hormones to migrate around the body and invoke the required reaction. By speeding up the system, waves of hormone releases initiated at different times would interfere, causing the responses invoked in destination cells to become out of sync with previous stages.

In the case of the electronic model, with each new input of data into the system, a new reaction cascades is initiated. With the variation in message migration time from source to target, data travelling around the system may end up being operated on in the wrong order.

The easiest solution is to allow the system to settle before starting a new reaction cascade. All hormones will have bound to cells or will have aged and have been removed from the system. This is of course a very inefficient solution as cells would spend the majority of time performing no function at all. It would be far better if cascades could occur simultaneously, each providing a chain of processing operations on consecutive input data. Thus allowing the data throughput to be vastly improved.

By incorporating a data input time stamp in each intercell message, cells are able to decipher between different message waves initiated by input data from different times. Cell receptors are open to messages with any time stamp, but the voting described in section 3.2 is taken only on messages with matching stamp values. A single cell is therefore able to concurrently deal with processing streams from different time steps. Overall, the pipeline of cell groups can remain filled, making much more efficient use of each cells processing time.

\subsection{Increasing Message Impact}

To help increase the impact a single message can have on a group of cells, the function of paracrine communication has been added to the model. As described in section 2 , paracrine communication involves local hormone distribution. It has the ability to reinforce the current activation trend of a neighbouring group of cells.

As considerably less inter-cell messages are to be used than hormones in endocrinology, the impact of a single message must be maximised. To accomplish this when cells receive a message destined for it, copies of the message are released to the cells direct neighbours, but are then stopped from travelling any further. As cells work in localised groups of like function, these neighbours are likely to also be sensitive to the copied hormones. As a result, the input voting stage of cells will receive the variety of messages it requires to invoke a reaction more quickly than be- 
fore this addition. The result is, a single message is capable of increasing the stimulation or inhibition level of a number of cells rather than just the one.

\subsection{Message Migration}

Hormones rely on the continual flow of blood around the body to help distribute themselves and to ensure that they reach their target. Similarly a process is required to help messages propagate around the cell network and avoid them from staying localised to one area. This is achieved by introducing a biasing in the choice of direction that hormones are transmitted in. In a boundary-less network, this has the effect of messages looping around the entire system, maximizing their cell coverage.

\section{The Bionode System}

The Bionode System was created to prove the suitability of an endocrinology inspired architecture as a reliability engineered processing platform. It was designed specifically to implement the model described in section 3 , however the highly reconfigurable nature of its structure makes it a useful development platform for implementing multinode systems.

A primary goal from the start of the project was to create a highly visual demonstrator that would show the robustness of the underlying system. To this end, rather than implement the whole system on a single PCB or chip, individual modules are used for each cell or node. This allows nodes to be physically removed whilst the system is operating and demonstrate its ability to tolerate the faults and recover from them.

The system contains thirty loosely coupled nodes in a boundary-less five by six grid. The toroid that is created by such a network is replicated by the physical structure of the system. Each node is connected to its nearest eight neighbours via a full duplex serial link. These links are also removable to provide an additional method of disrupting the systems operation. A picture of the bionode system is shown in figure 4.

\subsection{The Bionode}

Cells performing their role within the endocrine system undertake a number of tasks. Each bionode must be sufficiently flexible to model this. A single bionode, shown in figure 5, contains both a microcontroller and a FPGA. This simple combination provides a very powerful reconfigurable unit. The FPGA, a Xilinx Spartan XC2S100E, is used to implement the majority of the inter-node communication tasks. This leaves most of the processing time of the Atmel ATMega128L microcontroller available for operating on the data passing through the system; performing the function that system is implementing. Figure 6 shows a high-level block diagram of a single bionode module.

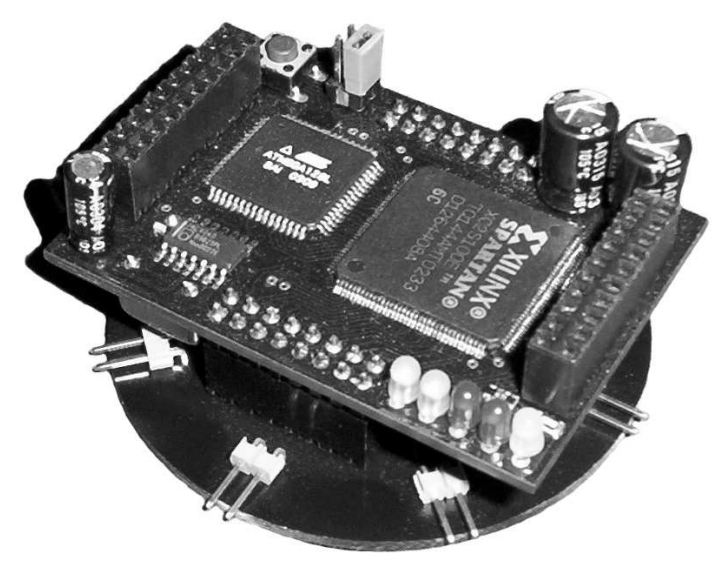

Figure 5. A single Bionode Module. The lower circular board holds the network interconnection points so that the Bionode board may be easily removed and replaced without disrupting the network wiring.

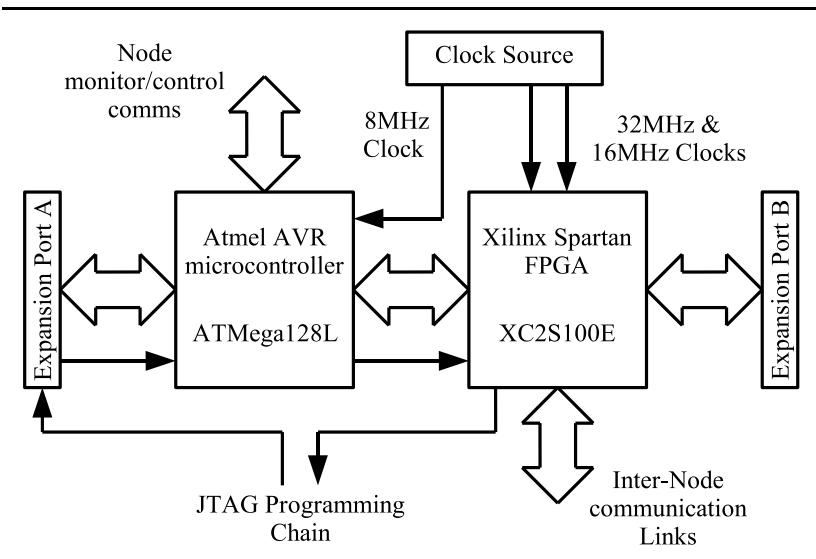

Figure 6. Block diagram of that parts that make up a single Bionode module.

\subsection{Inter-Node Communication}

The model that the bionode platform embodies is fundamentally a communication system. This being the case, 


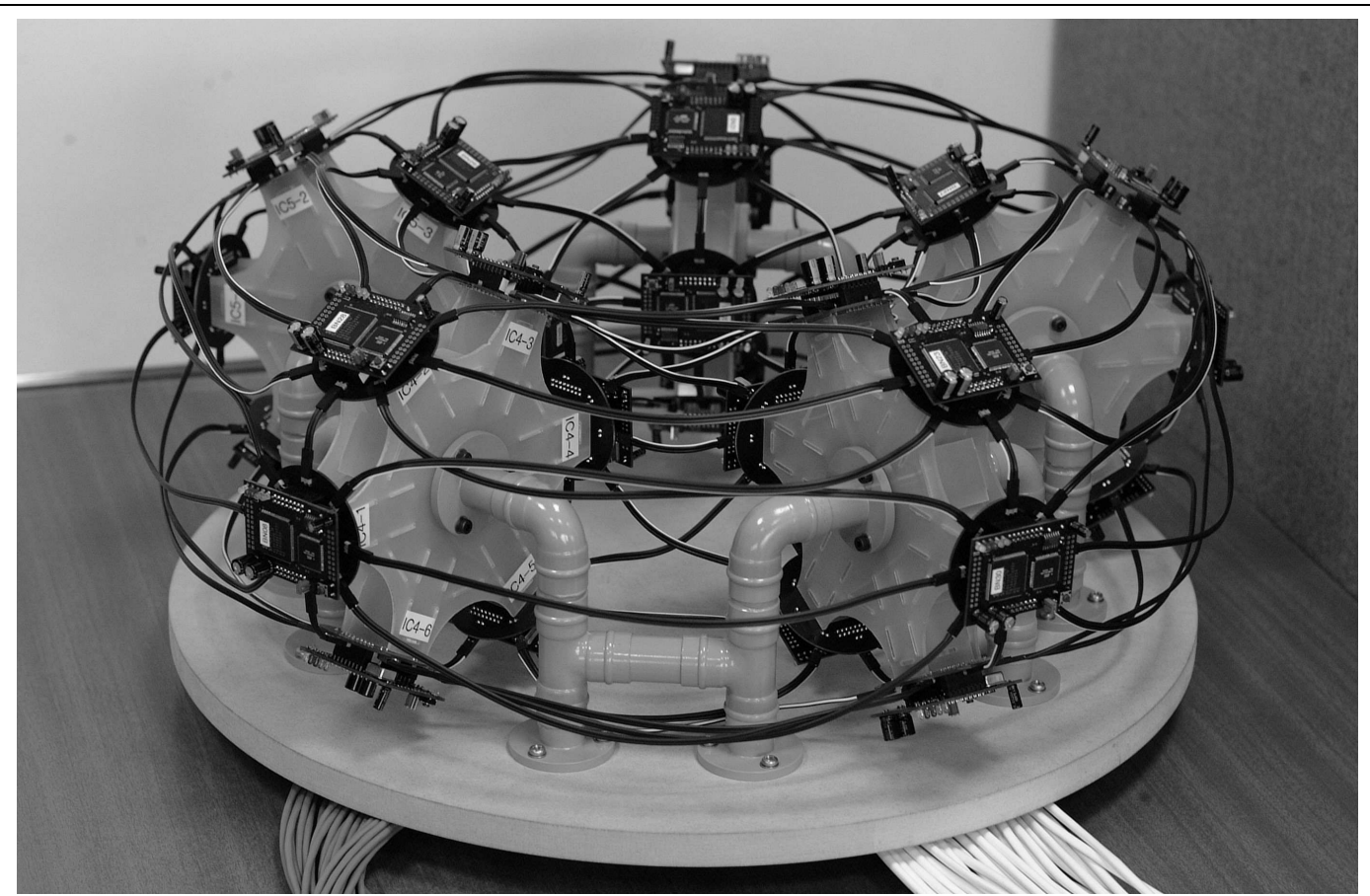

Figure 4. The Bionode toroid with thirty nodes. Also visible are the power and monitor cables.

creating an appropriate inter-node communication system is of utmost importance. The ability to easily disconnect nodes from the network could easily be compromised by using complex wiring between nodes. Therefore a simple two wire connection is used.

The circular interconnection board visible in figure 5 shows the connectors used for the inter-node links. Using a separate board just for creating the network infrastructure allows the node to be easily removed from the system without disrupting the network structure. With the future in mind, the sixteen communication links for each node are just as easily physically disconnected as they could be electronically in routing hardware if the entire bionode system was implemented on a single chip.

The communications protocol is asynchronous as there is no global clock signal between nodes. Inter-node messages are created from a string of individual serially encoded data packets. Supporting the 'hot-swapping' of nodes demands some interesting additions to the communication system. To stop the reception of packet segments causing receiver confusion, the data section of a packet is Manchester coded so the receiver can easily distinguish the non-encoded start and stop bits.

Similarly, so fragmented frames can be recognised as runts and not just short messages, the message layer of the communication protocol encapsulates the packet stream with a message header and footer. These both contain a matching id that can be used by the receiver to ascertain if a whole message has been received, half a message, or indeed two halves of different messages.

By reducing the inter-node link to two wires, busy signals are not immediately supportable. An extension of a technique used in the ethernet protocol [9] is used to solve this problem. Ethernet uses a link pulse transmitted at regular intervals to inform the destination of the source's presence. In the bionode system, this information is used to determine which of a nodes eight neighbours are available to receive a message. However, instead of a sending a simple pulse, a data packet is used. This packet updates the destination's records of the source's status. This information includes the receiver's buffer fill level, and thus allows a node to decide whether a connected node is able to accept data.

Moving to the receiving side of the communication stack message layer, to improve the speed that messages are able to migrate around the system, part of the message binding process is incorporated in the FPGA hardware. Messages that are not destined for a node are automatically marked for redirection. This saves the microcontroller from having to perform this task. Similarly, old messages that have travelled a defined number of hops between nodes are automatically removed from the system.

\subsection{Node Programming, Monitoring and Control}

Each bionode has a connection to a central control unit. However, this unit does not form any operational part of the 
bionode system, and therefore does not represent a single point weakness in the design. It is used purely to allow node configurations to be changed and to provide a monitor link for each bionode.

The monitor link is particularly important as it provides a means for status information to be passed back for analysis. It also provides another method for introducing faults into the system. Each node contains an individual JTAG chain that is used to program the microcontroller and FPGA. The advantage of using this type of scheme is the two connected devices can be programmed independently of each other, and the additional hardware costs are minimal.

\section{Conclusion}

Endocrinology incorporates a number of features that ensure its successful operation in the event of faults. It also operates within an architecture that allows simple cell removal and replacement. Consequently it is proving itself as a valid source of inspiration for an electronic system that exhibits similar reliability properties.

Many of the problems of converting a biological system into an architecture suitable for electronics have been solved. The problem of the unrealisable quantities of cells and hormones used in biology has been solved by increasing the area of effect inter-node messages have, and incorporating a voting system at the node message binding stage. The difference in biological operating speed and useful data processing speed has also been addressed. The solution makes efficient use of each cells available processing time by forming a pipeline effect of processing stages.

Simulations and models are useful tools for verifying designs, and measuring their chances of success if built into a real system. However they are not a substitute for the real systems constructed from those designs. With this in mind a novel hardware system has been built that is capable of implementing the endocrinology model.

Realising that inter-node communication is key to recreating the reliability of the endocrine system, a specialised communication stack has been created that allows messages to be quickly passed between nodes and whilst also supporting the 'hot-swapping' of those nodes. The bionode system also includes individual monitor access for each module so that the internal workings of the system may be analysed and used for confirmation of correct operation.

There is clearly a great deal of interesting work still to be done, some suggestions of courses that this may take are given in section 6 . However, it is believed that a useful hardware system can be created that is capable of performing arbitrary operations on data, in a manner that is tolerant to faults and is able to recover from them.

Further infomation and updates on the Bionode system may be found at http://www.bioinspired.com/ users/ajg112/projects/bionode/

\section{Further Work}

The Bionode hardware platform has only recently been completed. There are therefore many avenues of research still to explore with the project. Some of the more important directions are described here.

\subsection{Error Detection}

Clearly fault tolerance is only a single part of a reliability engineered system, leaving fault detection and repair. It is likely that the former will take inspiration from the biological immune system. Cells will be programmed to transform into immune system cells for a small proportion of their operation time. Taking on a role similar to a B-Cell, they will actively detect and check for faults in their neighbour cells.

\subsection{Self Reconfiguration}

Having achieved detection of faults it would be useful if the bionode system could do more than simply disconnect faulty nodes. It is planned that the system will start to make use of its built in redundancy and reorganise it self back to a full complement of functioning cell groups.

\subsection{Initial Self Organisation}

At the moment the bionode system needs priming at power-up with an initial configuration of cell functions. Although cells will need configuring with the correct data processing functions, ideally they would ascertain which function to perform and organise themselves into cell groups.

\subsection{Real World Application}

Producing a hardware system was an important first step in proving that the endocrine system can inspire a real world reliability engineered platform. The next step is to prove that the platform is capable of performing a real world task. The operation of the bionode system lends itself well to the area of Automated System Control. A suitable application in this field may be an implementation of a PID (Proportional-Intergral-Derivative) controller [6].

\section{References}

[1] C. Brook and N. Marshall. Essential Endocrinology. Blackwell Science, 4th edition, 2001.

[2] A. Greensted and A. Tyrrell. Fault tolerance via endocrinologic based communication. LNCS, Evolvable Systems: From Biology to Hardware, 2606:24-34, 2003. 
[3] C. Janeway, P. Travers, M. Walport, and J. Capra. Immuno Biology, The Immune System in Health and Disease. Garland Publishing, 4th edition, 1999.

[4] P. Lala. Fault Tolerant and Fault Testable Hardware Design. Prentice Hall, 1985.

[5] L. Nevitan, I.B. Kaczmarek. The Neuron: Cell and Molecular Biology. Oxford University Press, 3rd edition, 2002.

[6] N. Nise. Control Systems Engineering. Benjamin Cummings Publishing Company, 2nd edition, 1995.

[7] C. Ortega-Sanchez and A. Tyrrell. Design of a basic cell to construct embryonic arrays. IEE Transactions on Computers and Digital Techniques, 145-3:242-248, 1998.

[8] W. Shen, B. Salemi, and P. Will. Hormone inspired adaptive communication and distributed control for conro selfreconfi gurable robots. IEEE Transactions on Robotics and Automation, 18(5):700-712, 2002.

[9] C. Spurgeon. Ethernet: The Defi nitive Guide. O'Reilly, 2000.

[10] G. Tempesti, D. Roggen, E. Sanchez, and Y. Thoma. A poetic architecture for bio-inspired hardware. Proceedings of 8th International Conference on the Simulation and Synthesis of Living Systems, pages 111-115, 2002. 